\title{
Schmerzbehandlung beim Hochbetagten
}

Prognosen beschreiben einen Anstieg der Lebenserwartung auf 85 Jahre für Jungen und 88 Jahre für Mädchen bis 2060 in Deutschland. In 2060 würde sich dann der Anteil der über 67-Jährigen von jetzt 16\% auf $21 \%$ vergrößern. Patienten im höheren Lebensalter sind die am stärksten wachsende Bevölkerungsgruppe. Erwartungsgemäß einher gehen wird ein Anstieg des Bedarfes an entsprechenden Behandlungskapazitäten für spezifische Erkrankungen in höherem Alter, wie sie auch Schmerzerkrankungen darstellen. Trotz der Häufigkeit von Schmerzen bei Hochbetagten bleibt die Behandlung eine $\mathrm{He}$ rausforderung.

Zunächst wird in vielen Untersuchungen festgestellt, dass ältere Menschen Schmerzen häufiger hinnehmen und sie seltener als therapeutisches Ziel adressieren. Auf der einen Seite könnten weitere Erkrankungen mit ihren Symptomen und Auswirkungen im Vordergrund stehen, andererseits scheinen Patienten in höherem Alter häufiger eine Zunahme von Funktionseinschränkung und Schmerz als Auswirkung des Lebensalters per se anzunehmen. In der Folge werden chronische Schmerzen in dieser Lebensphase seltener ärztlich vorgestellt, häufig nicht erkannt oder nicht als behandlungsbedürftig wahrgenommen. Und dies insbesondere dann, wenn Patienten zusätzliche kognitive Einschränkungen aufweisen. Dagegen können immobilisierende Schmerzen eine grundsätzliche Einschränkung der Lebensqualität und Mobilität im Alter darstellen, schlussendlich sogar zu Rückzug und sozialer Isolation führen. Zudem besteht eine Interaktion von sozialem Rückzug und Einsamkeit sowie Schmerz. Vor diesem Hintergrund stellt die erste Säule der Schmerzbehandlung bei hochbetagten Patienten die Identifikation von akuten und chronischen Schmerzen dar.

Grundsätzlich lassen sich Schmerzen bei älteren Patienten nach ähnlichen Gesichtspunkten und Prinzipien behandeln, wie sie auch bei jüngeren Patienten etabliert sind. Gleichzeitig bestehen jedoch eine Reihe von Besonderheiten. Gut beschrieben ist die Tatsache, dass Medikamente sowohl in ihrer Wirkung als auch in ihrer Verarbei- tung durch den Körper bei älteren Patienten unterschiedlich sind. So wirken insbesondere sedierende Medikamente deutlich stärker in höherem Alter. Dies führt zwangsläufig zu einer höheren Inzidenz von unerwünschten Arzneimittelwirkungen wie z. B. Schwindel, Schläfrigkeit, Sedierung oder Störungen der Atmung sowie Stürzen. Auch die Wirkdauer und die Kinetik von Nebenwirkungen verläuft bei älteren Patienten anders; so könnten Medikamente bei längerer Einnahme kumulieren und dann zu schweren Komplikationen führen. Da sowohl die Leber- als auch die Nierenfunktion mit steigendem Alter typischerweise abnehmen, ist bei der Arzneimitteltherapie ein besonderes Fingerspitzengefühl gefragt. Der Blick in die Hausmedikation ist unerlässlich und bedarf einer aufmerksamen Evaluation hinsichtlich potenzieller Medikamenteninteraktionen. Hilfreich, wenn auch im Einzelfall nicht immer leicht umzusetzen, sind spezifische Listen gefährlicher Medikamente im Alter, wie sie z. B. in der PriscusListe veröffentlich sind. Schmerzen sind allerdings nicht nur mit Medikamenten zu behandeln.

Nicht-medikamentöse Verfahren bilden insgesamt eine wichtige Ergänzung - vor allem im Rahmen der Therapie von chronischen Leiden. Nicht selten sind sehr zeitund ressourcenintensive Interventionen notwendig, um lang bestehende Schmerzzustände zu behandeln. Eine zentrale Rolle spielt hier die Schmerzedukation, in die bei älteren Patienten Angehörige eingebunden werden sollten. Entsprechend fokussierte schmerz-psychotherapeutische Einrichtungen sind rar, von einer wohnortnahen Anbindung im ambulanten Bereich sind die meisten Regionen in Deutschland weit entfernt. Da die Verhaltenstherapie eine hohe Effektivität in der Schmerzreduktion aufweist und Achtsamkeit und positive Aufmerksamkeit fördert, verbessern sich häufig nicht nur Schmerzintensität, sondern auch Lebensqualität und Wohlbefinden. Wichtige Verfahren der Verhaltenstherapie sind dabei Entspannungstechniken wie die progressive Muskelentspannung nach Jacobson oder die Edukation zur Schmerzbewältigung. Weitere therapeutische Verfahren wie Physio- therapie, Ergotherapie oder spezifische Anwendungen wie die TENS sind nicht weniger wirksame Verfahren. Dennoch können hochbetagte Patienten mit Einschränkungen in der Mobilität, Kognition oder bei zunehmender Gebrechlichkeit schwieriger Termine in entsprechenden Einrichtung zur ambulanten physiotherapeutischen oder ergotherapeutischen Behandlung wahrnehmen. Auch die Anwendung von TENS in der Häuslichkeit bedarf oft Unterstützung. Insbesondere die nicht-medikamentösen Verfahren sind dabei im gegenwärtigen Fallpauschalensystem nicht adäquat abgebildet. Gerade zeitintensive Behandlungen, die Einbeziehung von Angehörigen und edukative Ansätze sind zumindest monetär für die Behandler weniger attraktiv. Hier könnte eine entsprechende Steuerung durch Gesetzgeber und Kostenträger gegenwirken.

Als problematisch erkannt ist andererseits, dass Studien zu hochbetagten Menschen ganz ähnlich wie bei nicht-einwilligungsfähigen Patienten und auch Kindern seltener für spezifische Fragestellungen zur Verfügung stehen. So ist die Evidenz der medizinischen Behandlung bei hochbetagten Patienten insgesamt geringer. Die Extrapolation von Studienergebnissen jüngerer Patienten auf hochbetagte Menschen muss vor dem Hintergrund der beschriebenen Unterschiede kritisch hinterfragt werden. Anreize für den Einschluss von älteren Patienten in Studien bestehen auf der anderen Seite zumindest für Sponsoren von wissenschaftlichen Untersuchungen eher nicht, vielmehr ist das Risiko immanent, bislang unerkannte Nebenwirkungen bei diesen Patienten zu identifizieren. Auch hier fehlen gegenwärtig spezifische Förderinstrumente für wissenschaftliche Studien an den medizinischen Hochschulen frei von Interessenskonflikten.

Ein konkretes Beispiel für die Entwicklung von chronischen Schmerzen besteht im Phantomschmerz, wie er zum Beispiel nach Amputation einer Extremität auftritt. Erfreulicherweise scheint durch den medizinischen Fortschritt bei immer weniger Patienten eine größere Extremitätenamputation notwendig zu sein. Gegenwärtig ist in Deutschland immer noch bei 
rund 55000 Patienten eine entsprechende Operation jährlich indiziert. Und wiederum sind vor allem ältere und hochbetagte Patienten betroffen, und zwischen 30$50 \%$ dieser Patienten entwickeln ein chronisches Schmerzsyndrom. Anders ausgedrückt: Allein für Deutschland ist von rund 20000 neuen Patienten mit Phantomschmerz pro Jahr auszugehen, der eine Behandlungsnotwendigkeit über Monate und Jahre nach sich ziehen kann.
Vor dem Hintergrund der deutlich steigenden Zahl älterer und hochbetagter Patienten in der Gesellschaft und im Gesundheitswesen sehen wir uns einer schwierigen, gemeinsamen Herausforderung gegenüber.

Interessenkonflikt

Die Autoren geben an, dass kein Interessenkonflikt besteht.

\section{Autorinnen/Autoren}

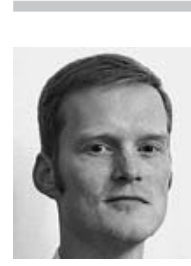

\section{Sascha Tafelski}

PD Dr. med., Oberarzt Anästhesiologie, Charité - Universitätsmedizin Berlin (CVK), Universitätskliniken für Anästhesiologie und operative Intensivmedizin

\section{Korrespondenzadresse}

PD Dr. Sascha Tafelski

Charité - Universitätsmedizin Berlin (CVK) Universitätskliniken für Anästhesiologie und operative Intensivmedizin Augustenburger Platz 1

13353 Berlin

sascha.tafelski@charite.de 